\title{
Dynamical behavior of two permutable entire functions
}

\author{
by Kin-Keung Poon and Chung-Chun Yang (Hong Kong)
}

\begin{abstract}
We show that two permutable transcendental entire functions may have different dynamical properties, which is very different from the rational functions case.
\end{abstract}

Introduction. During 1918-1926, Fatou and Julia initiated the theory of complex dynamics. They discovered the decomposition of the Riemann sphere into the sets now bearing their names (see, e.g., [3]-[5]).

Let $f(z)$ be a nonlinear entire function. The sequence of the iterates of $f$ is denoted by $f^{n+1}=f^{n} \circ f$, where $f^{0}=\mathrm{id}, f^{1}=f$. We define $F=F(f)$ to be the largest open set in the complex plane such that the sequence $\left\{f^{n}\right\}$ is normal there, and

$$
J=J(f)=\mathbb{C} \backslash F(f) .
$$

These sets are called the Fatou set and Julia set of $f$ respectively. From the definition, it can be verified easily that $F$ is open (possibly empty) and $J$ is closed. Moreover, $z \in J(f)$ if and only if for every neighborhood $U \ni z$ there is no convergent sequence $\left\{f^{n_{j}}\right\}$ on $U$.

Suppose that $U$ is a component of the Fatou set. Then $U$ is called a wandering domain if $f^{m}(U) \cap f^{n}(U)=\emptyset$ for $m \neq n$. If $U$ is not wandering, we call $U$ a pre-periodic component of $F(f)$. That is, $f^{n}\left(f^{m}(U)\right)=f^{m}(U)$ for $n, m \geq 0$. If $m=0$, we call $U$ a periodic component of $F(f)$.

As opposed to wandering domains, the behavior of $f^{n}$ on periodic components is well understood.

Let $U$ be a periodic component of period $p$. Then we have the following possibilities:

1. $U$ is called an attractive domain if $U$ contains an attracting periodic point $z_{0}$ of period $p$ and $f^{n p}(z)$ tends to $z_{0}$ for $z \in U$ as $n \rightarrow \infty$.

1991 Mathematics Subject Classification: Primary 30D05, 58F23.

Key words and phrases: entire function, complex dynamics, permutable functions.

The research was partially supported by a UGC grant of Hong Kong. 
2. $U$ is called a Leau domain if the boundary of $U$ contains an indifferent periodic point $z_{0}$ of period $p$ and $f^{n p}(z)$ tends to $z_{0}$ for $z \in U$ as $n \rightarrow \infty$.

3. $U$ is called a Siegel disc if there exists an analytic homeomorphism $\phi: U \rightarrow D$ where $D$ is the unit disc such that $\phi\left(f^{p}\left(\phi^{-1}(z)\right)\right)=e^{2 \pi i \alpha} z$ for some $\alpha \in \mathbb{R} \backslash \mathbb{Q}$.

4. $U$ is called a Herman ring if there exists an analytic homeomorphism $\phi: U \rightarrow A$ where $A$ is an annulus $\{z: 1<|z|<r\}$ for some $r>1$ such that $\phi\left(f^{p}\left(\phi^{-1}(z)\right)\right)=e^{2 \pi i \alpha} z$ for some $\alpha \in \mathbb{R} \backslash \mathbb{Q}$.

5. $U$ is called a Baker domain if there exists $z_{0} \in \partial U$ such that $f^{n p}(z) \rightarrow z_{0}$ for $z \in U$ as $n \rightarrow \infty$, but $f^{p}\left(z_{0}\right)$ is not defined. If $f$ is a transcendental entire function, then $z_{0}$ can only be infinity.

Julia [6] proved in 1922 that if two rational functions $f$ and $g$ commute, then $F(f)=F(g)$. The following problem remains open for nearly 80 years:

Problem 1. Given two permutable transcendental entire functions $f$ and $g$, i.e. $f \circ g=g \circ f$, does it follow that $F(f)=F(g)$ ?

Mathematicians not only want to solve this problem, but also to know more on the dynamical properties of two permutable functions. Dynamical properties here mean the nature and types of the components of the Fatou set according to the above classification. Schmidt [7] proved in 1992 that the dynamical properties of two permutable rational functions are very similar. Actually, he proved the following:

THEOREM A. Suppose $f$ and $g$ are two permutable rational functions. If a periodic component of $F(f)$ is an attractive domain, then it is also an attractive domain for $g$. Likewise, this result holds for a Leau domain, a Siegel disk and a Herman ring.

For transcendental entire functions, we have the following question.

Problem 2. Suppose $f$ and $g$ are permutable transcendental entire functions and $U$ is a common component of $F(f)$ and $F(g)$. Does $U$ have the same/similar dynamical properties for $f$ and $g$ ?

Besides providing some answers to Problem 2, we also investigate the Fatou sets of two permutable transcendental entire functions and answer Problem 1 affirmatively for certain classes of transcendental entire functions.

2. Lemmas and main results. As an improvement on Baker's result [1], we obtain the following.

LEMMA 2.1. Suppose $f$ and $g$ are permutable transcendental entire functions. If $g(z)=a f(z)+b$, where $a, b$ are constants and $a \neq 0$, then $F(f)=F(g)$. In addition, $|a|=1$. 
Lemma 2.2 (C. C. Yang and J. H. Zheng [8]). Let $f(z)=\sin z+Q(z)$, where $Q$ is a polynomial and $g$ is a nonlinear entire function of finite order, which is permutable with $f$. Then

1) if $\operatorname{deg} Q=0$, then $g=f$ or $g=-\sin z+k \pi, f=\sin z+k \pi$;

2) if $\operatorname{deg} Q=1$, then $g=f+2 k \pi$ or $g=-f+2 k \pi$ for some integer $k$;

3) if $\operatorname{deg} Q>1$ and $Q(z) \not \equiv-Q(-z)$, then $g=f$;

4) if $\operatorname{deg} Q>1$ and $Q(z) \equiv-Q(-z)$, then $g=f$ or $g=-f$.

Theorem 2.1. Suppose that $f(z)=\sin z+Q(z)$, where $Q(z)$ is a polynomial. If $g$ is a transcendental entire function of finite order which commutes with $f$, then $F(f)=F(g)$.

Basing on the above result, in Section 4 we construct an example to show that Problem 2 has a negative answer.

\section{Proofs of lemmas and theorems}

Proof of Lemma 2.1. We need only show that $F(f) \subset F(g)$ since then by symmetry we get $F(g) \subset F(f)$ and hence $F(f)=F(g)$. It is sufficient to prove $g(F(f)) \subset F(f)$ because then $\left\{g^{n}\right\}$ on $F(f)$ omits $J(f)$ which contains at least two points [2], so $\left\{g^{n}\right\}$ is normal on $F(f)$.

So suppose $g(F(f)) \subset F(f)$ is false. Take $\alpha \in F(f)$ with $g(\alpha) \notin F(f)$ and consider a neighborhood $U_{\alpha} \subset F(f)$ which contains $\alpha$. Consider any sequence $f^{n_{j}}$ convergent on $U_{\alpha}$. Then either $f^{n_{j}}$ converges to a holomorphic function $h: U_{\alpha} \rightarrow \mathbb{C}$ or to infinity (Hurwitz' theorem). In the former case $f^{n_{j}}$ converges to $g \circ h$ on $g\left(U_{\alpha}\right)$ since $f^{n} \circ g=g \circ f^{n}$. Therefore we have $g\left(U_{\alpha}\right) \subset F(f)$, a contradiction. So from now on we can assume $f^{n} \rightarrow \infty$ on $U_{\alpha}$. Therefore there exists an $n_{0}$ such that for $n \geq n_{0},\left|f^{n}(z)\right|>A$ for all $z \in U_{\alpha}$, where $A>(|b|+1) /|a|$. Thus for $n>n_{0}$,

$$
\left|f^{n} g(z)\right|=\left|g f^{n}(z)\right|=\left|a f^{n+1}(z)+b\right| \geq\left|a f^{n+1}(z)\right|-|b|>1 .
$$

Therefore for all $n>n_{0}$, the sets $f^{n}\left(g\left(U_{\alpha}\right)\right)$ are disjoint from the unit disc, so by the Montel theorem, the family $\left\{f^{n}\right\}$ is normal on $g\left(U_{\alpha}\right)$. This contradicts $g(\alpha) \notin F(f)$, hence we have the conclusion.

In order to prove $|a|=1$, note that $f \circ g(w)=g \circ f(w), g=a f+b$ and $f(w)=z$ give $f(a z+b)=a f(z)+b$. Let $a \neq 1$ and let

$$
z=y+\frac{b}{1-a} \quad \text { and } \quad h(y)=f\left(y+\frac{b}{1-a}\right)-\frac{b}{1-a} .
$$

Then

$$
f(a z+b)=f\left(a y+\frac{a b}{1-a}+b\right)=h(a y)+\frac{b}{1-a}
$$


and

$$
a f(z)+b=a f\left(y+\frac{b}{1-a}\right)+b=a h(y)+\frac{b}{1-a} .
$$

Hence we have $h(a y)=a h(y)$. If we consider the Taylor expansion of $h$, then

$$
a h(y)=\sum_{k=0}^{\infty} a h_{k} y^{k}
$$

and

$$
h(a y)=\sum_{k=0}^{\infty} h_{k} a^{k} y^{k} .
$$

By comparing the coefficients of (1) and (2), we have $h_{k}\left(a^{k}-a\right)=0$ for all $k$, hence

$$
a\left(a^{k-1}-1\right)=0 .
$$

Since $g$ is a transcendental entire function, we have $a \neq 0$. This implies that $|a|=1$.

Proof of Theorem 2.1. According to Lemma 2.2, $g$ is of the form $a f+b$, where $a, b$ are constants. Then by Lemma 2.1, we conclude $F(f)=F(g)$.

4. Counterexamples for Problem 2. Let $f(z)=-\sin z+z, g(z)=$ $-\sin z+z+2 \pi$, hence $g(z)=f(z)+2 \pi$. It is easy to check that $g \circ f=f \circ g$. By Lemma 2.1, $F(f)=F(g)$. For any integer $k$, we have $f(2 k \pi)=2 k \pi$ and $f^{\prime}(2 k \pi)=0$. Therefore $2 k \pi$ are attractive fixed points of $f$ for $k \in \mathbb{N}$. Let $U_{2 k \pi}$ be the attractive stable domain containing the attractive fixed point $2 k \pi$. Since $F(f)=F(g), U_{2 k \pi}$ is also a component of $F(g)$. Note that $U_{2 k \pi} \cap U_{2 k^{\prime} \pi}=\emptyset$ for $k \neq k^{\prime}$ and

$$
g(2 k \pi)=2 k \pi+2 \pi=2(k+1) \pi .
$$

On the other hand, since $2 k \pi \in U_{2 k \pi}$ and $2(k+1) \pi \in U_{2(k+1) \pi}$, we have

$$
g\left(U_{2 k \pi}\right)=U_{2(k+1) \pi} .
$$

Hence each $U_{2 k \pi}$ is a wandering domain of $g$.

From this example, we know that the dynamical behavior of two permutable transcendental functions can be very different, since we can find a common component $U$ in $F(f)$ and $F(g)$ such that it is an attractive domain of $f$ but a wandering domain of $g$.

Acknowledgements. We would like to thank the referee for his many valuable suggestions. 


\section{References}

[1] I. N. Baker, Wandering domains in the iteration of entire functions, Proc. London Math. Soc. (3) 49 (1984), 563-576.

[2] W. Bergweiler, Iteration of meromorphic functions, Bull. Amer. Math. Soc. 29 (1993), 151-188.

[3] P. Fatou, Sur les équations fonctionnelles, Bull. Soc. Math. France 47 (1919), 161271; 48 (1920), 33-94.

[4] —, Sur l'itération des fonctions transcendantes entières, Acta Math. 47 (1926), 337360 .

[5] G. Julia, Sur l'itération des fonctions rationnelles, J. Math. Pures Appl. (7) 4 (1918), 47-245.

[6] -, Sur la permutabilité des fractions rationnelles, Ann. Sci. Ecole Norm. Sup. 39 (1922), 131-215.

[7] W. Schmidt, On the periodic stable domains of permutable rational functions, Complex Variables Theory Appl. 17 (1992), 149-152.

[8] J. H. Zheng and C. C. Yang, Permutability of entire functions, Kodai Math. J. 15 (1992), 230-235.

Department of Mathematics

Hong Kong University of Science and Technology

Kowloon, Hong Kong

E-mail: mayang@uxmail.ust.hk

Reçu par la Rédaction le 17.2.1997

Révisé le 5.6.1997 